\title{
CXCL12-Mediated Regulation of ANP32A/Lanp, A Component of the Inhibitor of Histone Acetyl Transferase (INHAT) Complex, in Cortical Neurons
}

\author{
Muhammad Z. Khan • Avinash Vaidya • \\ Olimpia Meucci
}

Received: 8 March 2010 / Accepted: 22 June 2010 /Published online: 9 July 2010

(C) The Author(s) 2010. This article is published with open access at Springerlink.com

\begin{abstract}
The chemokine receptor CXCR4 and its endogenous ligand, CXCL12, are involved in development and homeostasis of the central nervous system and in the neuropathology of various neuroinflammatory/infectious disorders, including neuroAIDS. Our previous studies have shown that CXCR4 regulates cell cycle proteins that affect neuronal survival, such as the retinoblastoma protein, $\mathrm{Rb}$. These studies also suggested that Rb-mediated gene repression might be involved in the neuronal protection against NMDA exitotoxicity conferred by stimulation of the CXCL12/CXCR4 axis. In order to further test this hypothesis, we focused on the potential interaction of $\mathrm{Rb}$ with another protein implicated in regulation of gene expression, leucine-rich acidic nuclear protein (Lanp), also known as ANP32A/pp32/PHAP1. Lanp is a critical member of the protein complex inhibitor of histone acetyl transferase (INHAT), which prevents histone tail's acetylation, thus leading to transcriptional repression. Our data show that, in primary rat cortical neurons cultured for up to 30 days, Lanp is predominantly localized into the nucleus
\end{abstract}

Support: NIH-NIDA (R01-DA15014 and R01-DA19808 to OM)

Electronic supplementary material The online version of this article (doi:10.1007/s11481-010-9228-5) contains supplementary material, which is available to authorized users.

M. Z. Khan · A. Vaidya $\cdot$ O. Meucci $(\bowtie)$

Department of Pharmacology and Physiology, Drexel University College of Medicine,

245 N. 15th Street,

Philadelphia, PA 19102, USA

e-mail: omeucci@drexelmed.edu

O. Meucci

Department of Microbiology and Immunology, Drexel University

College of Medicine,

245 N. 15th Street,

Philadelphia, PA 19102, USA throughout the culture period, in line with in vivo evidence. Moreover, co-immunoprecipitation experiments show that endogenous Lanp interacts with $\mathrm{Rb}$ in neurons. Stimulation of CXCR4 by its endogenous ligand, CXCL12, increased Lanp protein levels in these neurons. Importantly, the effect of CXCL12 was preserved after exposure of neurons to NMDA. Finally, overexpression of exogenous Lanp in the neurons protects them from excitotoxicity. Overall, these findings suggest that Lanp can interact with $\mathrm{Rb}$ in both young and mature neurons and is implicated in the regulation of neuronal survival by CXCL12/CXCR4.

Keywords $\mathrm{Rb} \cdot \mathrm{CXCL} 12 \cdot$ cell survival $\cdot$ histone . chemokine $\cdot$ brain

\section{Introduction}

The chemokine receptor CXCR4 and its endogenous ligand CXCL12 are expressed constitutively in the central nervous system (CNS) during development and adulthood and play important roles in physiology and pathology ( $\mathrm{Li}$ and Ransohoff 2008). The CXCL12/CXCR4 axis has been implicated in important neuronal functions, such as modulation of neurotransmission, differentiation, and survival (Guyon and Nahon 2007; Li and Ransohoff 2008). Stimulation of CXCR4 by its natural ligand CXCL12 activates different signaling pathways in neurons ( $\mathrm{Li}$ and Ransohoff 2008). Previous studies from our group have shown that CXCR4 also controls specific cell cycle regulators such as p53 and the cdk-Rb-E2F pathway in postmitotic neurons (Khan et al. 2005; Shimizu et al. 2007; Khan et al. 2008). These proteins may be involved in transcriptional regulation of specific sets of genes that affect the survival of postmitotic neurons independently of their 
role in cell cycle (Becker and Bonni 2004). In line with this, our findings indicate that CXCR4 positively regulates $\mathrm{Rb}$ and this controls the expression of E2F1 pro-apoptotic targets in neurons, thus resulting in neuroprotection (Khan et al. 2003; Shimizu et al. 2007; Khan et al. 2008). Interestingly, non-physiological ligands of CXCR4 that induce abnormal stimulation of CXCR4, such as the HIV-1 envelope protein gp120 $0_{\text {IIIB }}$, are unable to stimulate $\mathrm{Rb}$ function and induce neurotoxicity via E2F-dependent pathways (Shimizu et al. 2007; Bachis et al. 2009). This further supports the role of $\mathrm{Rb}$ in neuroprotection and highlights the importance of proper function of the CXCL12/CXCR4 axis in the CNS.

$\mathrm{Rb}$ is able to bind more than 100 different proteins, some of which directly or indirectly control cell fate (Morris and Dyson 2001). These interactions determine the various specific roles of $\mathrm{Rb}$ in a cell. $\mathrm{Rb}$ mostly acts as a gene suppressor and interacts with well-known transcription factors (e.g., E2F1), as well as enzymes regulating the post-translational modifications of histones (Macaluso et al. 2006). These chromatin modifications favor chromatin condensation, thus inhibiting gene expression by limiting access of transcription complexes to the DNA. Among the $\mathrm{Rb}$-interacting proteins that regulate transcriptional activity, ANP32a/Lanp (also known as pp32, PHAP1, I1PP2A; henceforth referred to as Lanp in this paper) is also found in neurons (Matsuoka et al. 1994; Adegbola and Pasternack 2005). It belongs to a family of leucine-rich repeatscontaining proteins (Matilla and Radrizzani 2005). Lanp is an integral component of the inhibitor of histone acetyl transferase complex (INHAT), which blocks transcription by masking $\mathrm{N}$-terminal histone tails from the activity of histone acetyl transferases or through interaction with histone deacetylases (Seo et al. 2001; Seo et al. 2002). Recent studies indicate that, through its INHAT activity, Lanp is involved in both physiological and pathological processes in the CNS (Matilla and Radrizzani 2005). One example is its ability to regulate expression of neurofilament genes in neurons, which affects neuronal differentiation (Kular et al. 2009). Furthermore, alterations of Lanp functions have been implicated in spinocerebellar ataxia type 1 (SCA1) and Alzheimer's disease (Matilla et al. 1997; Chen et al. 2008). However, the precise role of Lanp in neuronal survival is still unclear. Furthermore, while its interaction with $\mathrm{Rb}$ prevents cell death (Adegbola and Pasternack 2005), studies in proliferating cells indicate that Lanp can also be involved in apoptosis (Jiang et al. 2003; Pan et al. 2009).

Our earlier studies demonstrate that $\mathrm{Rb}$ plays a central role in the neuroprotective functions of CXCL12/CXCR4 (Khan et al. 2008). Given the reported interaction of Lanp and $\mathrm{Rb}$ in non-neuronal cells (Adegbola and Pasternack 2005), we asked whether they may also interact in neurons supporting their survival. Here, we examined the constitutive and CXCL12-stimulated expression of endogenous Lanp in primary neuronal cultures at different stages of differentiation, its interaction with $\mathrm{Rb}$, and the effect of Lanp overexpression on excitotoxicity. Our data show for the first time a protective role of this nuclear $\mathrm{Rb}$ partner in postmitotic neurons.

\section{Materials and methods}

Neuronal cultures Cortical neurons were obtained from the brains of 17-day-old rat fetuses and cultured in a serum-free medium using the bilaminar cell culture system (Banker and Cowan 1977; Kaech and Banker 2006), in which pure neuronal cultures are grown in the presence of a separate glial feeder layer supporting their growth and differentiation. This model reproduces, to a certain extent, the in vivo environment (i.e., the presence of neuronal and nonneuronal cells) and offers the major advantage that neurons can be separated from the glia at any time in order to analyze neuron-specific biological responses. We have extensively used the bilaminar culture system to study expression and function of chemokine receptors in neurons, including CXCR4, and previously demonstrated that the signaling pathways induced by direct activation of neuronal CXCR4 promote neuronal survival mechanisms, even in the absence of glia (Khan et al. 2004; Sengupta et al. 2009). In these studies, unless otherwise noted, CXCL12 treatments $(20 \mathrm{nM})$ were performed in the presence of glia in order to predict the potential outcome of CXCL12 stimulation in vivo. Neurons were always separated from the glia immediately before protein extraction. Cortical neurons were plated on poly-L-lysine-coated $15-\mathrm{mm}$ coverslips $\left(40 \times 10^{3}\right.$ or $50 \times 10^{3}$ cells $)$ or 60 -mm dishes $\left(1 \times 10^{6}\right.$ cells) - depending on the type of experiment. Treatments started after at least 7 days in vitro (7 DIV).

Immunocytochemistry Neurons were fixed in $4 \%$ paraformaldehyde. For neuronal marker staining, anti- $\beta$ tubulin III (clone TUJ1, 1:500, from Covance, Berkeley, CA, USA) was used, followed by a cy3-conjugated (Fig. 1) or a cy2conjugated secondary antibody (Fig. 5a). For Lanp staining, anti-Lanp (C-18, 1:200, Santa Cruz Biotechnology, Santa Cruz, CA, USA) was followed by a biotin-conjugated secondary antibody and then by cy3-streptavidin. To visualize FLAG-Lanp-expressing neurons (Fig. 5a), a DYKDDDDK Tag antibody that detects FLAG (cat\# 2368, 1:800, Cell Signaling Technology, Danvers, MA, USA) was used followed by a cy3-conjugated secondary antibody (Fig. 5a). Nuclear staining was obtained using Hoechst $33342(0.5 \mu \mathrm{l} / \mathrm{ml})$. Cells were observed under an epifluorescent microscope (Olympus IX70, Melville, NY, 
Fig. 1 Expression of Lanp in primary cortical neurons: Neurons were cultured for $1-$ 30 days as reported in the methods section, then fixed and stained for endogenous Lanp (green) using a specific antibody. The neuronal marker $\beta$ tubulin III (red) was used for visualization of neuronal cell bodies while the nuclei were stained with Hoechst 33342 (blue). Scale bar $=50 \mu \mathrm{m}$
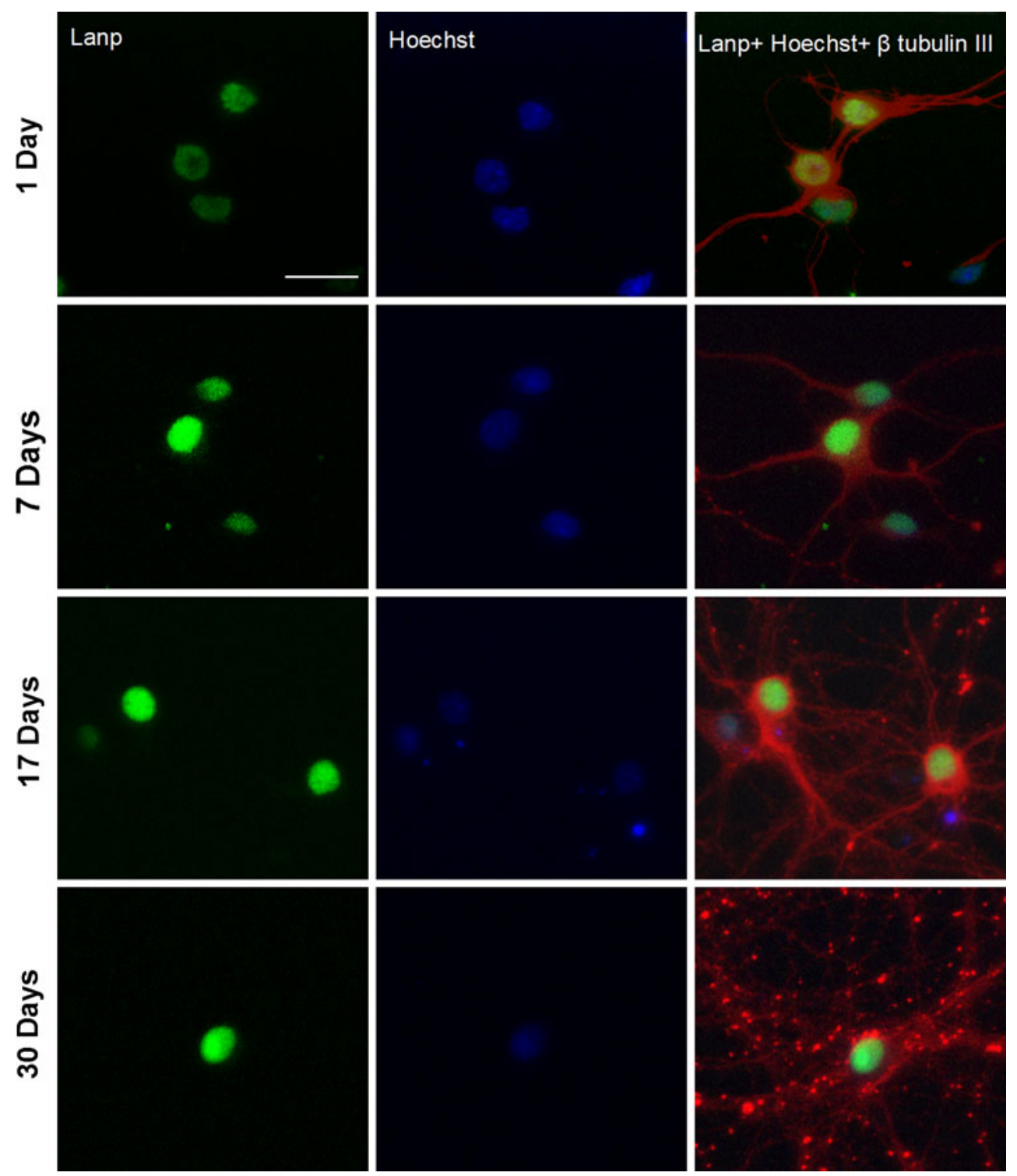

USA) connected to a CCD camera (Micromax, Trenton, NJ, USA), and images were taken using Metamorph software (Molecular Devices, Sunnyvale, CA, USA).

Western blots For total cell lysates, neurons were scraped in lysis buffer $(25 \mathrm{mM}$ Tris, $150 \mathrm{mM} \mathrm{NaCl}, 5 \mathrm{mM} \mathrm{NaF}, 1 \mathrm{mM}$ EDTA, $1 \mathrm{mM}$ DTT, 1\% IGEPAL-CA-630, $5 \mathrm{mg}$ each of aprotinin, leupeptin, and pepstatin, $1 \mathrm{mM}$ AEBSF, and $1 \mathrm{mM}$ vanadate), as reported previously (Shimizu et al. 2007; Khan et al. 2008). The protein concentration of cell lysates was determined using the bicinchoninic acid assay from Pierce (Rockford, IL, USA). Equal amounts of proteins $(25 \mu \mathrm{g})$ were loaded in each lane, separated by SDS-PAGE and transferred to PVDF membranes for immunoblotting. The following primary antibodies were used: anti-Lanp (C-18, 1:1,000; Santa Cruz Biotechnology), anti-Rb (1:4,000,G3-245; BD Biosciences PharMingen,
Franklin Lakes, NJ, USA), and anti-Actin (1:5,000, A2066; Sigma-Aldrich, St. Louis, MO, USA). Bands were detected by chemiluminescence using the Pierce SuperSignal West Femto maximum sensitivity kit, according to the manufacturer's instructions, and analyzed using the FluorChem 8900 apparatus from Alpha Innotech (San Leandro, CA, USA).

Immunoprecipitation For immunoprecipation (IP), $250 \mu \mathrm{g}$ protein was used for each treatment (Fig. 3). IP reactions were performed with goat anti-Lanp or monoclonal anti-Rb antibodies and ExactaCruz ${ }^{\mathrm{TM}} \mathrm{B}$ (sc-45039), or ExactaCruz $^{\mathrm{TM}}$ A reagents (sc-45038, Santa Cruz Biotechnology), respectively, were used, according to the manufacturer's protocols. The precipitated extracts were immunoblotted and probed for Lanp and $\mathrm{Rb}$ proteins as described above. 
Survival assays and transfections For survival assays, rat cortical neurons $\left(50 \times 10^{3}\right.$ cells per coverslip) were divided in two groups. One group was transfected with pFLAG-Lanp vector (expressing murine Lanp; a gift from Dr. Huda Zoghby, Baylor College of Medicine, Houston, TX, USA), whereas the other group was transfected with a control plasmid expressing GFP; the Lipofectamine 2000 reagent (Invitrogen, Carlsbad, CA, USA) was used for transfection (4 $\mu \mathrm{g}$ of plasmid per coverslip). NMDA neurotoxicity assay was conducted as described before (Khan et al. 2008); only the cells expressing GFP and those positive to FLAG immunostaining were counted (Fig. 5). Transfection efficiency in these experiments was about $35 \%$.

Materials Unless otherwise specified, tissue culture media are from Invitrogen. CXCL12 (SDF-1 $\alpha$ ) was purchased from PeproTech (Rocky Hill, NJ, USA); the chemokine was reconstituted in $\mathrm{PBS} / 0.1 \% \mathrm{BSA}$, stored as stock solution at $-20^{\circ} \mathrm{C}$, and used at a final concentration of $20 \mathrm{nM}$. AMD3100 was obtained from Sigma-Aldrich, reconstituted in water, and used at final concentrations of $100 \mathrm{ng} / \mathrm{ml}$.

Statistical analysis Student's $t$ test or ANOVA (followed by Newman-Keuls multiple comparison test) were used for statistical analysis $(P<0.05$ was considered statistically significant). All data are reported as mean \pm SEM. Experiments were performed at least three times.

\section{Results}

Expression of Lanp protein in cortical neurons and its regulation by the chemokine CXCL12 Recent ex vivo studies have shown that Lanp is expressed predominantly in the neuronal nuclei in distinct regions of the brain (Kovacech et al. 2007). However, earlier studies in neuronal cell lines have suggested the possibility of subcellular translocation (i.e., from nucleus to cytosol) of Lanp upon differentiation (Opal et al. 2003). In order to determine intracellular localization of Lanp in primary neuronal cultures, immunostaining of Lanp was performed on rat cortical neurons cultured for up to 4 weeks (Fig. 1). At all the time periods studied (from 1 to 30 days), Lanp was found to be expressed mainly in the neuronal nuclei, and no increase in the cytosolic levels of the protein was observed upon neuronal differentiation and maturation at any time (Fig. 1). Thus, Lanp expression in our primary cultures reflects the in vivo situation; these data also suggest that the expression of the protein in neuronal cell lines may be altered and/or influenced by the cell cycle.
Our previous studies indicate that CXCR4 is able to control $\mathrm{Rb}$ transcriptional activity (Khan et al. 2008). We have shown that RNAi-mediated $\mathrm{Rb}$ depletion in cortical neurons leads to the disruption of CXCR4-mediated protection against neurotoxicity (Khan et al. 2008). Lanp is known to interact with $\mathrm{Rb}$, and this interaction is implicated in the regulation of cell survival in different cell lines (Adegbola and Pasternack 2005). To determine whether the CXCL12/ CXCR4 axis can also regulate Lanp protein levels, differentiated rat cortical neurons were treated with CXCL12 (20 nM for $30 \mathrm{~min}$ ) and protein extracts collected after $3-5 \mathrm{~h}$-in analogy to our studies with $\mathrm{Rb}$. Western blot analysis showed an increase in the endogenous Lanp protein in treated neurons (Fig. 2a). Pre-treatment with the CXCR4antagonist, AMD3100 $(100 \mathrm{ng} / \mathrm{ml}$, added to the culture medium before CXCL12), abolished the up-regulation of Lanp by CXCR4, confirming the involvement of CXCR4 activity in this effect (Fig. 2a). Overall, these data indicate that CXCR4 stimulation regulates Lanp in cortical neurons.

Interaction of Lanp with $\mathrm{Rb}$ and its involvement in CXCL12 neuroprotection In order to determine whether Lanp interacts with $\mathrm{Rb}$ in neurons, IP studies were performed in cortical neuronal extracts using specific antibodies against Lanp or $\mathrm{Rb}$. Immunoprecipitates were then probed by Western blots for both of these proteins individually (Fig. 3). These experiments indicate that endogenous $\mathrm{Rb}$ and Lanp co-immunoprecipitate, suggesting that they constitutively interact in neurons (Fig. 3). Next, we asked whether Lanp may be involved in the CXCL12 neuroprotective effect along with $\mathrm{Rb}$; based on our previous studies concerning the regulation of Rb by CXCL12 and its consequences on neuronal survival (Khan et al. 2008), we predicted that CXCL12 would be able to up-regulate Lanp levels in control, as well as NMDA-treated, neurons. To test this hypothesis, neurons were treated with CXCL12 and/or NMDA (100 $\mu \mathrm{M}$ for $20 \mathrm{~min})$ as we previously reported (Khan et al. 2008). Cell extracts were collected after $5 \mathrm{~h}$, immunoblotted, and probed for Lanp. As expected, CXCL12 was able to increase Lanp protein levels in both control and NMDA-treated neurons (Fig. 4).

Role of Lanp in neuroprotection against NMDA exitotoxicity To study the role of Lanp in neuronal survival, we used a Lanp expression vector (Fig. 5a), in which the Lanp sequence is fused with FLAG tag at N-terminal (Matilla et al. 1997). Expression of this vector in primary cortical neurons led to Lanp localization to the nucleus similar to endogenous Lanp (Fig. 5a). Moreover, Flag-Lanp did not affect cell survival under basal conditions (Fig. 5b), in contrast to findings in proliferating cells (Jiang et al. 2003; Pan et al. 2009). To explore if Lanp could protect neurons from excitotoxicity, neurons transfected with the same 

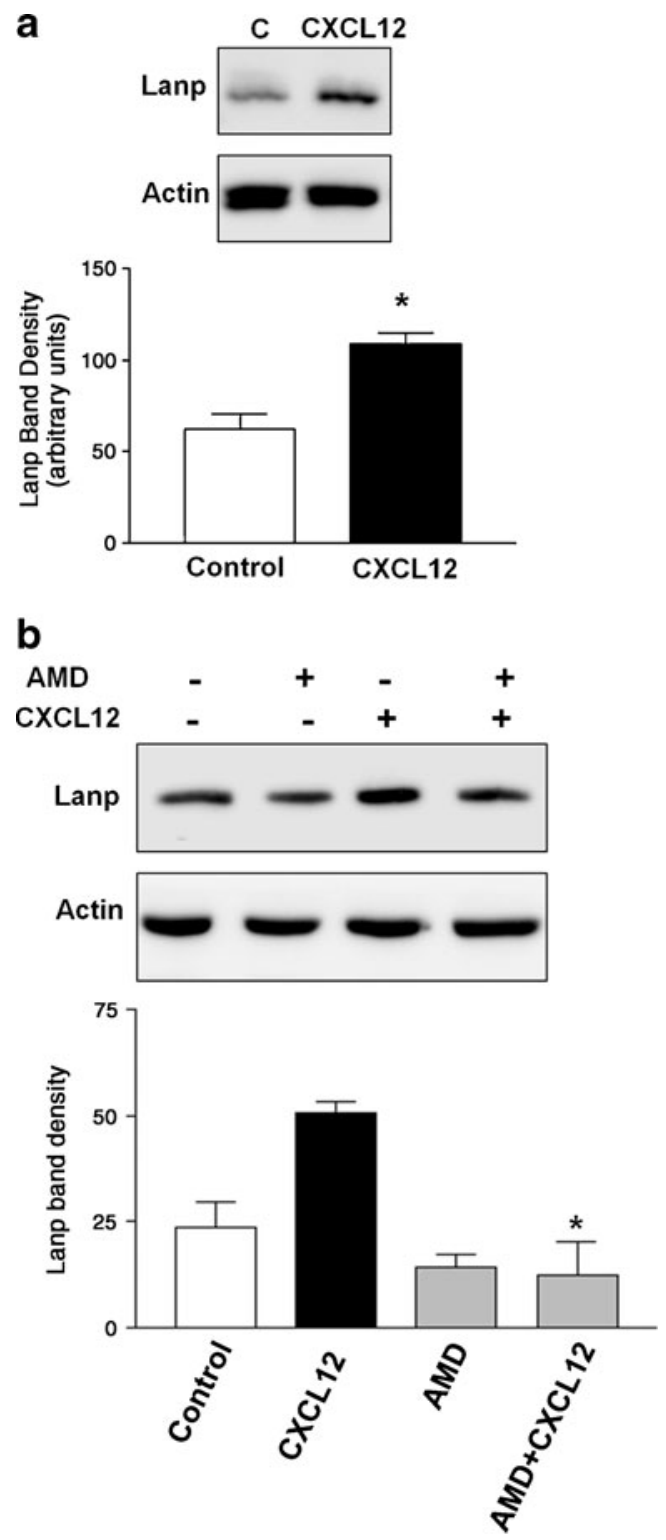

Fig. 2 CXCL12 increases endogenous Lanp levels in cortical neurons: Rat cortical neurons were treated with CXCL12 $(20 \mathrm{nM})$ for $30 \mathrm{~min}$; total cell extracts were collected at 3-5 h after treatment and immunoblotted for Lanp and actin (a). Equal amounts of protein $(25 \mu \mathrm{g})$ were loaded in each lane. Densitometric analysis of three independent experiments is reported in the graph (A) as mean $\pm \mathrm{SEM}$ of band density units (asterisk, $P<0.05$ vs control; $n=3$ ). The immunoblots on the bottom (B) are from additional studies with neurons treated with the specific CXCR4 antagonist, AMD 3100 $(100 \mathrm{ng} / \mathrm{ml})$. AMD was added to the culture $15 \mathrm{~min}$ before exposure to CXCL12 (20 nM, $5 \mathrm{~h})$; at the end of the treatment, total cell lysates were extracted and immunoblotted for Lanp and actin. The graph (B) represents the densitometric analysis of three independent experiments (asterisk, $P<0.05$ vs CXCL12)

vector were treated with NMDA (100 $\mu \mathrm{M}$ for $20 \mathrm{~min})$ and neuronal death assessed $24 \mathrm{~h}$ later; a set of control neurons transfected with a GFP expression vector that underwent the same NMDA treatment was used for comparison. In our previously published studies, this NMDA protocol has shown
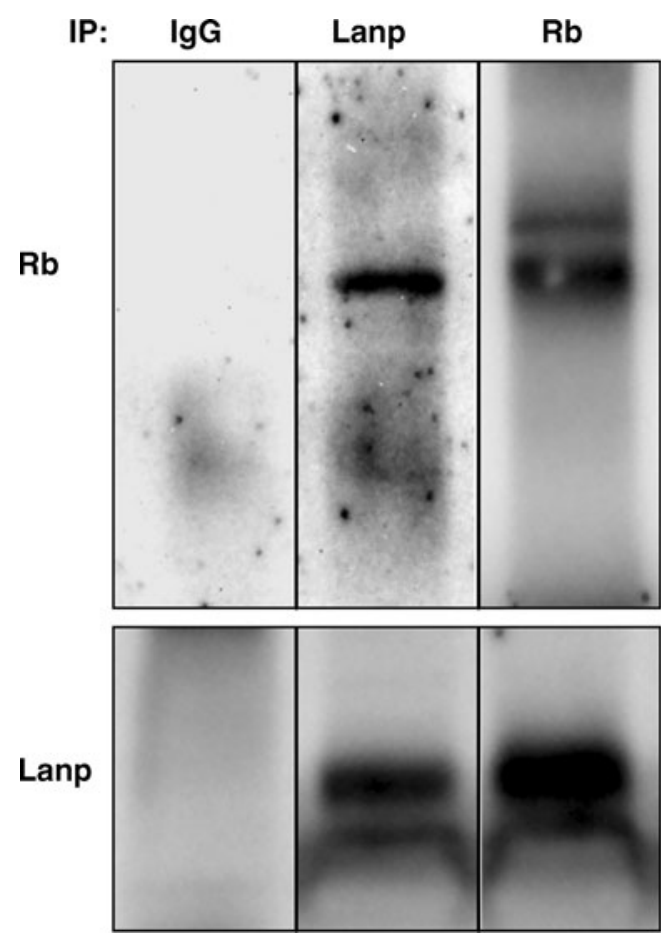

Fig. 3 Endogenous Lanp interacts with $\mathrm{Rb}$ in neurons: Equal amounts of neuronal extracts $(250 \mu \mathrm{g}$; 9DIV) were incubated with either anti-Rb or anti-Lanp before electrophoresis/immunoblotting for both $\mathrm{Rb}$ and Lanp. A specific $\mathrm{IgG}$ mixture was used as immunoprecipitation control

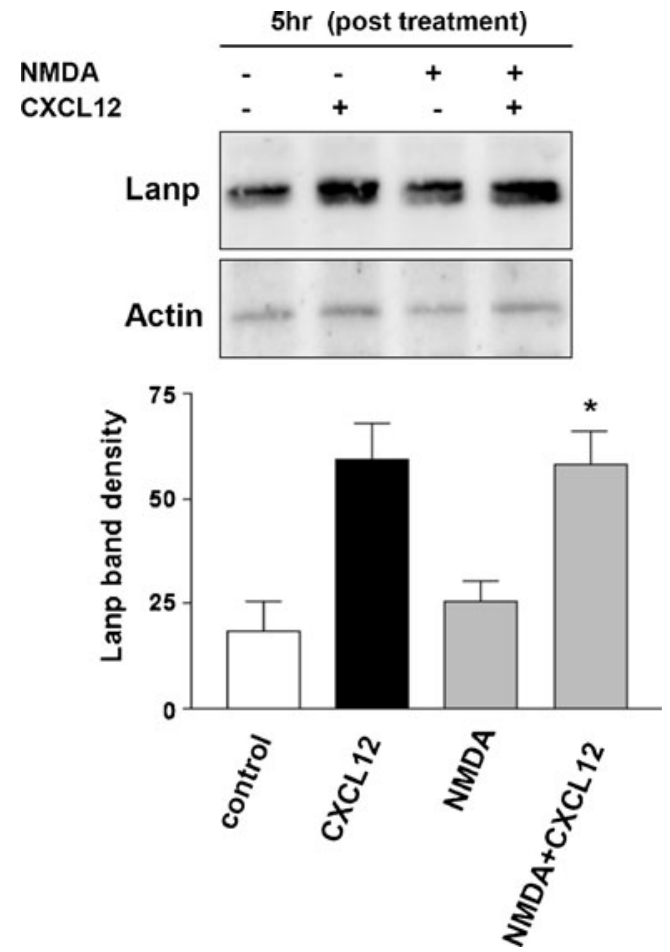

Fig. 4 Effect of CXCL12 and NMDA on neuronal Lanp: Vehicle- or CXCL12-treated neurons were stimulated with NMDA $(100 \mu \mathrm{M}$ for $20 \mathrm{~min}$ ); neuronal extracts were collected after $5 \mathrm{~h}$ and immunobotted for Lanp and actin. Densitometric analysis of three independent experiments is reported in the graph as mean $\pm \mathrm{SEM}$ of band density units (asterisk, $P<0.05$ vs NMDA; $n=3$ ) 


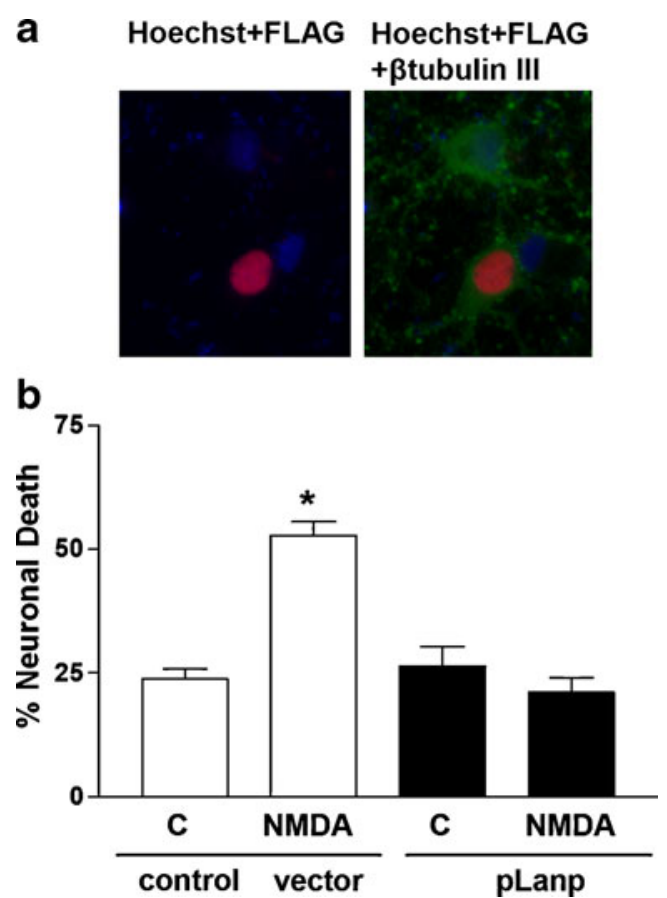

Fig. 5 Overexpression of Lanp protects neurons from NMDA toxicity: Neurons were transfected with a Flag-Lanp expression vector (pFlagLanp). The exogenous Lanp was visualized by antiFLAG antibody (a). Cells were also stained for a neuronal marker, $\beta$ tubulin III (green) and Hoechst 33342 to visualize nuclei (blue). Scale $b a r=50 \mu \mathrm{m}$. Neurons transfected either with control pGFP vector or pLanp vector were treated with NMDA $(100 \mu \mathrm{M}, 20 \mathrm{~min})$ and fixed after $24 \mathrm{~h}$ to determine cell survival. FLAG-LANP-expressing neurons were visualized by immunocytochemistry and data collected from three independent experiments is expressed as mean \pm SEM in the graph (b). Asterisk, $P$ vs NMDA (pLanp) $<0.001$

to induce $60 \%$ to $70 \%$ neuronal death (Patel et al. 2006). As opposed to NMDA-treated GFP-expressing cells, Lanpexpressing cells were significantly protected from NMDA neurotoxicity $(P<0.001)$. This is the first evidence of a protective role of Lanp in neurons.

\section{Discussion}

This report lends support to the role of CXCL12/ CXCR4 axis in the control of neuronal survival and adds Lanp to the list of modulators of gene expression that are regulated by CXCR4. Furthermore, our data suggest that Lanp might be involved in CXCL12/ CXCR4 neuroprotection from excitotoxicity (Fig. 6). Lanp belongs to a family of evolutionarily conserved proteins that have leucine-rich repeats in their N-termini (Matilla and Radrizzani. 2005). These motifs are involved in its interaction with a variety of other proteins, and these interactions determine the range of its functions in a specific cell type. Its acidic C-terminus contains the nuclear localization signal (Pan et al. 2009).
In this report, we observe that, in primary cortical neurons, native Lanp is expressed predominantly and abundantly in the nucleus throughout neuronal differentiation. Thus, the subcellular translocation of Lanp as shown by an earlier study in a differentiating neuronal cell line seems to be limited to that specific cell line, since all other evidence, including this paper, argues against it being a common mechanism in CNS (Opal et al. 2003; Kovacech et al. 2007). Interestingly, previous studies in proliferating cells have shown that cytosolic Lanp is involved in pathways that affect cell survival. For example, cytosolic Lanp is involved in granzyme A-induced death of cytotoxic T-lymphocyte target cells, or, in other cells, it is a part of a specific complex that promotes formation and function of apoptosome resulting in caspase-mediated cell death (Fan et al. 2003; Jiang et al. 2003; Pan et al. 2009). On the contrary, in postmitotic neurons, Lanp is primarily expressed in the nucleus, can interact with other endogenous nuclear proteins, such as $\mathrm{Rb}$, and promote neuronal survival. Lanp is an essential part of the INHAT complex and, as a result, it is involved in binding to and, thus, masking the N-terminal histone tails from histone acetyl transferase activity (Seo et al. 2001; Seo et al. 2002). Hence, Lanp acts mainly as a gene repressor. Recent studies in neuronal cells indicate that Lanp interacts with a transcriptional repressor, E4F, and enhances its activity, further supporting the nuclear role for Lanp in neurons (Cvetanovic et al. 2007). Moreover, in the context of neurodegenerative disorders, the mutated ataxin-1, which has been implicated in spinocerebellar ataxia type 1 (SCA1), is able to relieve Lanp-E4F gene repression by competing with Lanp for binding to E4F; these interactions might lead to SCA1 neuropatholgy in cerebellum (Cvetanovic et al. 2007). The present study shows that the chemokine CXCL12 positively regulates neuronal Lanp. Considering the interaction of endogenous Lanp with $\mathrm{Rb}$, these data suggest that Lanp might be

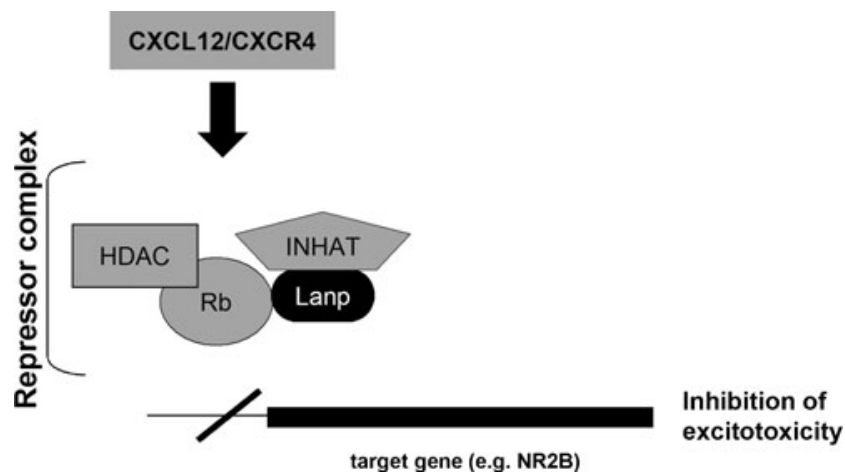

Fig. 6 A schematic diagram showing the CXCL12/CXCR4-mediated signaling events leading to the putative formation of Lanp-Rb gene repressor complex and its effect on neuronal survival 
involved in CXCR4-regulated $\mathrm{Rb}$ signaling in neurons. Our previous studies implicate $\mathrm{Rb}$ and $\mathrm{Rb}$-mediated gene repression in the neuroprotection action of CXCL12 (Khan et al. 2008). Novel data also show that CXCL12 reduces in vitro/in vivo expression of specific neuronal genes, namely, the NR2B subunit of NMDA receptor, normally controlled by HDAC/Rb-dependent mechanisms (Nicolai et al. 2010; Qiu and Ghosh 2008). As a result of the limited expression of NR2B, CXCL12 would be able to control calcium entry via extrasynaptic NMDA receptor, thus preventing excitotoxicity (Nicolai et al. 2010). The findings reported in the present paper suggest that Lanp might be one of the $\mathrm{Rb}$-interacting proteins involved in neuronal survival in response to NMDA insult.

In agreement with this conclusion, when Lanp is overexpressed in cortical neurons, it retains its nuclear localization and protects neurons against NMDA toxicity. Overall, these results suggest that Lanp acts as an Rbinteracting protein in neurons and may play a significant role in neuronal survival in vivo. These data are in line with other reports showing that Lanp regulates the promoter of the neurofilament light chain (NF-L) gene through inhibition of histone acetylation, and it is thus implicated in neurite outgrowth (Kular et al. 2009). Interestingly, CXCR4 stimulation leads to comparable effects during CNS development and differentiation (Pujol et al. 2005). It is possible that Lanp mediates some of these effects. Unfortunately, we were unable to provide direct evidence about this in the present study as depletion of Lanp in these neurons by shRNA resulted in major alterations of neuronal differentiation, in agreement with and in addition to the previous studies (Kular et al. 2009; Suppl. Figs. 1 and 2). Thus, NMDA-induced neurotoxicity was negligible in these cultures (not shown). Further investigations using conditional knockout of Lanp or alternative approaches may be necessary to address this issue.

In conclusion, our results show that CXCL12 positively regulates nuclear expression of Lanp, which may act in concert with $\mathrm{Rb}$ to ultimately reduce excitotoxicity (Fig. 6). Although further studies are required to determine the molecular mechanisms involved in the regulation of Lanp by CXCR4 and its direct link to neuroprotection, our data raise the possibility that Lanp, along with $\mathrm{Rb}$, contributes to suppression of specific genes that play a critical role in neuronal survival and differentiation.

Conflicts of interest The authors declare no conflicts of interest.

Open Access This article is distributed under the terms of the Creative Commons Attribution Noncommercial License which permits any noncommercial use, distribution, and reproduction in any medium, provided the original author(s) and source are credited.

\section{References}

Adegbola O, Pasternack GR (2005) Phosphorylated retinoblastoma protein complexes with pp 32 and inhibits pp32-mediated apoptosis. J Biol Chem 280:15497-15502

Bachis A, Biggio F, Major EO, Mocchetti I (2009) M- and T-tropic HIVs promote apoptotis of rat neurons. J Neuroimmune Pharmacol 4:150-160

Banker GA, Cowan WM (1977) Rat hippocampal neurons in dispersed cell culture. Brain Res 126:397-425

Becker EB, Bonni A (2004) Cell cycle regulation of neuronal apoptosis in development and disease. Prog Neurobiol 72:1-25

Chen S, Li B, Grundke-Iqbal I, Iqbal K (2008) I1PP2A affects tau phosphorylation via association with the catalytic subunit of protein phosphatase 2A. J Biol Chem 283:10513-10521

Cvetanovic M, Rooney RJ, Garcia JJ, Toporovskaya N, Zoghbi HY, Opal P (2007) The role of LANP and ataxin 1 in E4F-mediated transcriptional repression. EMBO Rep 8:671-677

Fan Z, Beresford PJ, Oh DY, Zhang D, Lieberman J (2003) Tumor suppressor NM23-H1 is a granzyme A-activated DNase during CTL-mediated apoptosis, and the nucleosome assembly protein SET is its inhibitor. Cell 112:659-672

Guyon A, Nahon JL (2007) Multiple actions of the chemokine stromal cell-derived factor-1alpha on neuronal activity. J Mol Endocrinol 38:365-376

Jiang X, Kim HE, Shu H, Zhao Y, Zhang H, Kofron J, Donnelly J, Burns D, Ng SC, Rosenberg S, Wang X (2003) Distinctive roles of PHAP proteins and prothymosin-alpha in a death regulatory pathway. Science 299:223-226

Kaech S, Banker G (2006) Culturing hippocampal neurons. Nat Protoc 1:2406-2415

Khan MZ, Brandimarti R, Musser BJ, Resue DM, Fatatis A, Meucci O (2003) The chemokine receptor CXCR4 regulates cell-cycle proteins in neurons. J Neurovirol 9:300-314

Khan MZ, Brandimarti R, Patel JP, Huynh N, Wang J, Huang Z, Fatatis A, Meucci O (2004) Apoptotic and antiapoptotic effects of CXCR4: is it a matter of intrinsic efficacy? Implications for HIV neuropathogenesis. AIDS Res Hum Retroviruses 20:1063-1071

Khan MZ, Shimizu S, Patel JP, Nelson A, Le MT, Mullen-Przeworski A, Brandimarti R, Fatatis A, Meucci O (2005) Regulation of neuronal P53 activity by CXCR 4. Mol Cell Neurosci 30:58-66

Khan MZ, Brandimarti R, Shimizu S, Nicolai J, Crowe E, Meucci O (2008) The chemokine CXCL12 promotes survival of postmitotic neurons by regulating $\mathrm{Rb}$ protein. Cell Death Differ 15:1663-1672

Kovacech B, Kontsekova E, Zilka N, Novak P, Skrabana R, Filipcik P, Iqbal K, Novak M (2007) A novel monoclonal antibody DC63 reveals that inhibitor 1 of protein phosphatase $2 \mathrm{~A}$ is preferentially nuclearly localised in human brain. FEBS Lett 581:617-622

Kular RK, Cvetanovic M, Siferd S, Kini AR, Opal P (2009) Neuronal differentiation is regulated by leucine-rich acidic nuclear protein (LANP), a member of the inhibitor of histone acetyltransferase complex. J Biol Chem 284:7783-7792

Li M, Ransohoff RM (2008) Multiple roles of chemokine CXCL12 in the central nervous system: a migration from immunology to neurobiology. Prog Neurobiol 84:116-131

Macaluso M, Montanari M, Giordano A (2006) Rb family proteins as modulators of gene expression and new aspects regarding the interaction with chromatin remodeling enzymes. Oncogene 25:5263-5267

Matilla A, Radrizzani M (2005) The Anp32 family of proteins containing leucine-rich repeats. Cerebellum 4:7-18

Matilla A, Koshy BT, Cummings CJ, Isobe T, Orr HT, Zoghbi HY (1997) The cerebellar leucine-rich acidic nuclear protein interacts with ataxin-1. Nature 389:974-978 
Matsuoka K, Taoka M, Satozawa N, Nakayama H, Ichimura T, Takahashi N, Yamakuni T, Song SY, Isobe T (1994) A nuclear factor containing the leucine-rich repeats expressed in murine cerebellar neurons. Proc Natl Acad Sci USA 91:9670-9674

Morris EJ, Dyson NJ (2001) Retinoblastoma protein partners. Adv Cancer Res 82:1-54

Nicolai J. Burbassi S, Rubin J, Meucci O (2010) CXCL12 inhibits expression of the NMDA receptor's NR2B subunit through a histone deacetylase-dependent pathway contributing to neuronal survival. Cell Death and Disease 1, e33 (1 April 2010) doi:10.1038/cddis.2010.10

Opal P, Garcia JJ, Propst F, Matilla A, Orr HT, Zoghbi HY (2003) Mapmodulin/leucine-rich acidic nuclear protein binds the light chain of microtubule-associated protein $1 \mathrm{~B}$ and modulates neuritogenesis. J Biol Chem 278:34691-34699

Pan W, da Graca LS, Shao Y, Yin Q, Wu H, Jiang X (2009) PHAPI/pp 32 suppresses tumorigenesis by stimulating apoptosis. J Biol Chem 284:6946-6954

Patel JP, Sengupta R, Bardi G, Khan MZ, Mullen-Przeworski A, Meucci O (2006) Modulation of neuronal CXCR4 by the microopioid agonist DAMGO. J Neurovirol 12:492-500
Pujol F, Kitabgi P, Boudin H (2005) The chemokine SDF-1 differentially regulates axonal elongation and branching in hippocampal neurons. J Cell Sci 118:1071-1080

Qiu Z, Ghosh A (2008) A calcium-dependent switch in a CRESTBRG1 complex regulates activity-dependent gene expression. Neuron 60:775-787

Sengupta R, Burbassi S, Shimizu S, Cappello S, Vallee RB, Rubin JB, Meucci O (2009) Morphine increases brain levels of ferritin heavy chain leading to inhibition of CXCR4-mediated survival signaling in neurons. J Neurosci 29:2534-2544

Seo SB, McNamara P, Heo S, Turner A, Lane WS, Chakravarti D (2001) Regulation of histone acetylation and transcription by INHAT, a human cellular complex containing the set oncoprotein. Cell 104:119-130

Seo SB, Macfarlan T, McNamara P, Hong R, Mukai Y, Heo S, Chakravarti D (2002) Regulation of histone acetylation and transcription by nuclear protein pp 32, a subunit of the INHAT complex. J Biol Chem 277:14005-14010

Shimizu S, Khan MZ, Hippensteel RL, Parkar A, Raghupathi R, Meucci O (2007) Role of the transcription factor E2F1 in CXCR4-mediated neurotoxicity and HIV neuropathology. Neurobiol Dis 25:17-26 